\title{
Tangos y sambas (in)apropiados: ocio de resistencia
}

\author{
Tangos and sambas (in)adequates: resistance leisure
}

Tangos e sambas (in)apropriados: ócio de resistência

\author{
JoSe MANUEl Alvarez SEARA ${ }^{1}$ \\ UNIVERSIDAD DE LA REPÚBLICA, UDELAR, MALDONADO, URUGUAY
}

\begin{abstract}
RESUMEN
Las danzas de salón tango y samba gafieira son expresiones en las que se manifiesta la hegemonía de la heteronormatividad en las cuales se legitiman ciertos estereotipos de género. El presente trabajo indaga sobre las resistencias, re-negociaciones y alternativas que existen en estas danzas a esa hegemonía heteronormativa. La metodología de este trabajo es cualitativa, configurándose como estudio de caso, en el que nos proponemos observar y analizar los diferentes discursos de personas que trabajan con las danzas de salón mencionadas, realizando entrevistas en profundidad, observaciones participantes y triangulando estas observaciones con la teoría de género y la teoría queer. Los resultados de este trabajo apuntan las diversas resistencias a ciertos estereotipos de género y en el análisis y observación de la danza como lenguaje en la cual pueden ser exhibidos discursos y performances de personas que desafían la heteronormatividad hegemónica.
\end{abstract}

Palabras clave: Danza. Heteronormatividad. Ocio. Género. Queer.

\begin{abstract}
The tango and samba gafieira dances are expressions in which the hegemony of heteronormativity is manifested in which certain gender stereotypes are legitimized. The present work inquiries about the resistances, renegotiations and alternatives that exist in these dances to that heteronormative hegemony. The methodology of this work is qualitative, case study, in which we propose to observe and analyse the different discourses of people who work with the room dances mentioned above, conducting in-depth interviews, participant observations and triangulating these observations with gender theory and queer theory. The results appoint collect the different resistances to certain gender stereotypes, and in the analysis and observation of dance as a language, the inscriptions and speeches of people who question hegemonic heteronormativity can be visualized. Keywords: Dance. Heteronormativity. Leisure. Gender. Queer.
\end{abstract}

\section{RESUMO}

As danças de salão tango e samba gafieira são expressões em que se manifesta a hegemonia da heteronormatividade, em que os estereótipos de gênero são legitimados. O presente trabalho indaga sobre as resistências, renegociações e alternativas que existem nessas danças a essa hegemonia heteronormativa. A metodologia deste estudo é qualitativa, configurando-se como estudo de caso, em que nos propomos a observar e analisar os diferentes discursos das pessoas que trabalham com as danças de salão mencionadas, realizando entrevistas, observações participantes e triangulando essas observações com a teoria do gênero e a teoria queer. Os resultados apontaram várias resistências a certos estereótipos de gênero e a análise e observação da dança como uma linguagem na qual podem ser exibidos discursos e performances de pessoas que desafiam a heteronormatividade hegemônica.

Palavras-chave: Dança. Heteronormatividade. Ócio. Gênero. Queer.

\footnotetext{
${ }^{1}$ Profesor adjunto del Departamento de Tiempo Libre, Ocio y Educación Física, Instituto Superior Educación Física, Centro Universitario Regional Este, UdelaR. E-mail: josmanu3@gmail.com
} 


\section{INTRODUCCIÓN}

El presente artículo académico es parte de mi investigación de doctorado, que estoy llevando a cabo bajo la dirección de Dra. María Jesús Monteagudo Sánchez, en el programa de Ocio, Cultura y Comunicación para el Desarrollo Humano de la Universidad de Deusto, España. En donde observe clases y bailes de tango y samba gafieira en espacios públicos y privados de tres ciudades latinoamericanas (Buenos Aires, Argentina; Montevideo, Uruguay; São Paulo, Brasil).

Argentina, Uruguay y Brasil tienen leyes de respeto de los derechos de las personas Lésbicas, Gays, Bisexuales, Transexuales, Intersexuales y Queer (LGBTIQ), en el caso de Uruguay y Argentina ambos países han sido referentes en la América Latina en relación a las leyes que consagran el matrimonio igualitario, el cambio de identidad y la posibilidad de adopción de niñas y niños sin importar el sexo biológico.

Sin embargo, se pueden observar ciertos estereotipos de género que aún persisten en la sociedad, en el caso de la cultura, en el tango y samba gafieira específicamente existen ciertos estereotipos de género que predominan, estos son creencias que se van sedimentando a través de los años y se pueden manifestar que fueron absorbidos y/o apropiados por los países en una determinada época como símbolos de la cultura nacional (GARRAMUÑO, 2009).

Las danzas de tango y samba gafieira son danzas de salón que se tornaron ritmos nacionales, la primera de Argentina y Uruguay, en cuanto la segunda de Brasil (GARRAMUÑO, 2009). Por otro lado las letras de tango tienen un gran poder de crear ficciones que producen ciertos imaginarios (GASPARRI, 2011). Saikin (2004) realiza una exhaustiva análisis de esas letras para desentrañar estereotipos, y roles de género mayoritariamente heteronormativos, donde el tango reproduce un machismo bárbaro que coloca al hombre por sobre la mujer, donde muchas veces ese dominio se manifiesta de forma violenta y que puede ser traducido como violencia de género.

Conviene subrayar que, sin dejar de lado la música y las letras que acompañan a estos estilos musicales, el objeto principal de este trabajo de investigación es la danza indagando sobre las resistencias, re-negociaciones y alternativas que existen en estas danzas de salón a esa hegemonía heteronormativa.

Fort i Marrugat (2015) subraya que desde una perspectiva de género, existe en la danza una masculinidad hegemónica que se transmite mediante valores que reproducen estereotipos de género en los que predominan tópicos según los cuales, los hombres deben expresar masculinidad y las mujeres feminidad (FORT I MARRUGAT, 2015).

En relación con el tango y con el samba gafieira se pueden observar en los últimos años los movimientos de tango queer/gay/milongas libres en las ciudades de Buenos Aires, Montevideo y São Paulo que proponen un quiebre con ciertos estereotipos de género. Como manifiesta provocativamente Cecconi (2009), podría hablarse metafóricamente que la danza de tango queer existe desde sus comienzos en Buenos Aires a fines del siglo XIX cuando se bailaba en el territorio del arrabal donde era bailado por hombre-hombre, hombre-mujer y mujer-mujer. Cecconi (2009) va a releer la historia del tango y hacer esa afirmación, que puede estar acertada de manera metafórica, porque como bien manifiesta la autora el movimiento queer es un movimiento que surge en Buenos Aires a finales de los años 1990. Pero la metáfora es usada para afirmar, y es algo con lo que concordamos, que en el inicio del tango no existía una sola manera de bailar esta danza, no existía, se podría decir, una única manera de bailar esta danza. Asimismo en relación al samba gafieira, en São Paulo se observan lugares donde se puede aprender a bailar samba gafieira sin una restricción heteronormativa. 
La hegemonía heteronormativa provoca ciertas discriminaciones en la danza donde las personas que no se adaptan a esa matriz son, en algunos casos, estigmatizadas, consideradas como desviadas (BASSETTI, 2013), y/o son llamados, por ejemplo, como afeminados o gays los hombres (RISNER, 2007) y las mujeres como marimachos (BASSETTI, 2013).

Tomando a la danza como expresión de ocio y como manifiesta Shaw (2001), como ocio de resistencia, que puede modificar y sobreponerse a la opresión que viven las personas, en este caso la heteronormatividad en la danza, este trabajo indaga sobre esas resistencias que promueven cambios, empoderamientos y cuestionamientos en la danza de tango y samba gafieira heteronormativa.

\section{METODOLOGía}

El diseño metodológico utilizado es cualitativo, estudio de caso (COLLER, 2000), con la combinación de diferentes métodos que son la revisión documental, el análisis del discurso (FOUCAULT, 1990), la realización de entrevistas semiestructuradas, observaciones participantes (WACQUANT, 2006; CORBETTA, 2007) y la triangulación con teorías de género y queer, en donde se observaron cómo se reinterpretan ciertos estereotipos de género heteronormativos (BUTLER, 2002) en la danza de tango y samba gafieira.

Se propuso para el trabajo de campo realizarlo en tres ciudades latinoamericanas, Buenos Aires, Montevideo y São Paulo, ciudades de tres países limítrofes, respectivamente: Argentina, Uruguay y Brasil, en las que vive la mayor proporción de habitantes de cada país. Esta demarcación arbitraria está dada en relación a que en estas ciudades existe una mayor práctica de estas danzas de salón, representadas por la cantidad de escuelas de danza, locales de baile privados y espacios públicos donde se desarrollan clases y bailes.

Se realizaron 6 entrevistas en profundidad (CORBETTA, 2007), 2 en cada ciudad, a personas que trabajan en las danzas mencionadas como profesores y profesoras. Se utilizó el método de bola de nieve (ATKINSON; FLINT, 2001) para poder encontrar a las personas y realizar las entrevistas.

Las entrevistas en profundidad nos van a servir, para la reconstrucción de acciones pasadas, el estudio de las representaciones sociales (estereotipos, normas) y las interacciones que se observan entre las personas (DELGADO; GUTIÉRREZ, 1999).

En el presente trabajo está realizado conforme a la ley orgánica 15/1999 de España, donde se protegen los datos de las personas entrevistadas, garantizando su confidencialidad. Asimismo las personas firmaron un consentimiento libre y esclarecido, se hace la aclaración que van a aparecer como referencia en este trabajo bajo la denominación persona entrevistada y un número y sus participaciones van a ser expresadas literalmente sin traducciones o correcciones gramaticales.

También hicimos un total de 28 observaciones participantes (WACQUANT, 2006) de clases y bailes, donde 10 observaciones fueron en Buenos Aires, 8 en Montevideo y 10 en São Paulo. A su vez la muestra fue subdividida en espacios públicos y espacios particulares/privados.

La observación participante ejecuta su tarea de investigación desde dentro de las realidades humanas que pretende abordar. Es el principal instrumento de trabajo de la etnografía, por ejemplo, y apoyo para generar las impresiones del investigador que son registradas en el llamado diario de campo. Se trata de un registro continuo y acumulativo de todo lo que sucede y es captado por el investigador (CORBETTA, 
2007).

Para las observaciones participantes se construyeron fichas de observación en las que se consideraron las variables de vestimenta, estereotipos de género, las resistencias de las personas a los estereotipos de género, y las estrategias que utilizan para sobrepasar y/o renegociar dichos estereotipos.

Mi participación como investigador también está presente desde poner el cuerpo, ya que también bailo y soy participe de las actividades de las danzas que estoy investigando (WACQUANT, 2006).

\section{DANZA DE SALÓN Y HETERONORMATIVIDAD}

Para Yamanashi y Bulman (2007), las danzas de salón tomaron notoriedad en los Estados Unidos en los años 1990, cuando programas de shows televisivos como Dancing with the stars, mostraron a personas que competían bailando. Esos programas mostraban ciertos estereotipos de género en los que las mujeres siempre eran conducidas por los hombres, donde el papel de líder siempre era el del hombre, que se puede relacionar con los estudios de género y queer, en que se asignan según el sexo biológico de la persona ciertos roles de género a las personas. En los Estados Unidos se observan ciertas actitudes femeninas y masculinas adjudicadas según su sexo biológico, para los autores durante el siglo 18, 19 y 20, los estadunidenses percibían a las mujeres como lindas, delicadas e indefensas y a los hombres como líderes y protectores.

Según Yamanashi y Bulman (2007), las danzas de salón reflejan una sociedad tradicional en que la mujer sea pasiva, elegante y frágil, donde se reflejan la libertad del hombre que tiene que proteger, guiar y dar seguridad financiera a la mujer.

Asimismo Risner (2002) afirma que existen en la cultura estadunidense preconceptos en relación a los hombres que bailan, ellos son llamados de gays/afeminados, se sedimentan estereotipos de masculinidad y homofobia en los que los hombres deben jugar fútbol y/o todos los deportes, pero no bailar (RISNER, 2002). Esa comparación de la virilidad masculina asociada a los deportes es algo que autores como Risner (2002) y Gard (2001) mencionan, una construcción que se mantiene hasta hoy en los ballets de danza y el ejemplo es el modelo de hip-hopper para los hombres que habla Bassetti (2013). Además se observa que los hombres ocupan lugares reconocidos y centrales en escuelas, academias y compañías de danza (BASSETTI, 2013).

Las danzas de tango y samba tienen una matriz de género en que se refuerza una cierta masculinidad asociada al liderazgo y el poder (CAMACHO, 2000) que son construidos socialmente.

Según Berocan (2012) la danza de gafieira es una expresión de civilización, que es regulada por el autocontrol, la elegancia de los pasos y en el que la mujer es llevada por el hombre conforme a construcciones sociales de género bien definidas.

Como manifiesta Magali Saikin (2004) en el caso del tango, es un lugar donde las relaciones de género ocupan un lugar relevante y esas relaciones son más variadas que las normalmente conocidas y divulgadas. En este sentido se naturalizaron históricamente roles en la danza que fueron adaptados a los sexos biológicos, pero nuevas investigaciones históricas (SAIKIN, 2004; CECCONI, 2009; HAVMOELLER; BATCHELLOR; ARAMO, 2015) vienen a cuestionar el hecho de que la danza de tango fuera siempre heteronormativa, y en sus trabajos ponen en duda la heteronormatividad en la danza de tango desde sus inicios. 


\section{RESISTENCIAS DE OCIO A LA HETERONORMATIVIDAD EN LAS DANZAS DE SALÓN}

Preciado (2008) va a criticar la construcción que algunas personas hacen sobre la heterosexualidad y va a decir que no ha existido siempre. Butler (2002) va a hablar de la heteronormatividad, que está asociada al sexo biológico, y se expresa en un binarismo hombre-mujer. Para la autora la categoría sexo es un principio normativo, que significa para la autora el mismo que Foucault llamó de ideal regulatorio en sus trabajos. Para Butler (2002) el sexo no sólo funciona como norma, sino que también es una práctica reguladora que produce los cuerpos que gobierna, cuya fuerza se manifiesta como una especie de poder productivo, capaz de demarcar, circunscribir y diferenciar los cuerpos que controla.

De Lauretis (1990) va a decir que el género es una construcción sociocultural, que está atravesada por representaciones discursivas y visuales, que pueden ser observadas en diferentes instituciones y también de forma menos evidente en el lenguaje, el arte, la literatura y el cine.

Tomando como relevante los estudios de género y queer vamos a indagar en el lenguaje discursivo y corporal de las danzas de tango y samba gafieira para poder visualizar esa construcción sociocultural de género que se configura como hegemonía heteronormativa y de cómo las personas resisten, re-negocian y buscan alternativas a esa hegemonía.

El tango y samba gafieira pareciera se constituyen como una tradición inventada (HOBSBAWM; RANGER, 2012) en relación a ciertos códigos y estereotipos de género. Existe también cierta discriminación en las milongas tradicionales de tango y bailes tradicionales de samba gafieira de poder bailar hombre-hombre y mujer-mujer en las tres ciudades investigadas. Existen diferentes tipos de discriminación, que van desde la vestimenta hasta la prohibición de bailar hombre con hombre, así como mujer con mujer.

En uno de los locales de baile, posiblemente uno de los más emblemáticos de samba gafieira de Brasil, La estudiantina, en Río de Janeiro, se pueden observar carteles donde se deja explícito y se hace manifiesto diferentes tipos de discriminación como son la prohibición de bailar personas del mismo sexo biológico, así como reglamentación de la vestimenta, en los estatutos de gafieira (BEROCAN, 2012).

En Buenos Aires existieron hasta hace pocos años carteles de discriminación y prohibición de bailar para las personas del mismo sexo, que tuvieron que ser retirados por una nueva ley antidiscriminatoria de la ciudad ${ }^{2}$.

Maslak y Votruba (2011) manifiestan que la danza como expresión artística tiene una tradición que se sustenta en cuestiones locales y normas, que están asentadas en el patriarcado. Como manifiesta una de las personas entrevistadas "[...] yo creo que el tango surge desde ese lugar, surge como una apropiación machista" (PERSONA ENTREVISTADA 4).

Maslak y Votruba (2011) afirman que esas tradiciones y códigos de la danza de tango se expresan de diferentes formas, por un lado en el código de que la invitación a bailar es por parte de los hombres que se llama cabeceo ${ }^{3}$ y por otro lado como

\footnotetext{
2 BUENOS AIRES. Ley $5261 . \quad$ Disponible en: http://www2.cedom.gov.ar/es/legislacion/normas/leyes/ley5261.html >. Accedido en: 30 oct. 2018.

${ }^{3}$ Cabeceo es la acción que se realiza con la cabeza y se utiliza para invitar a bailar a una persona en un baile de tango, mayoritariamente es realizado el cabeceo por los hombres hacia las mujeres (MASLAK; VOTRUBA, 2011), lo que genera que las mujeres inevitablemente están siempre atentas observando a los hombres y esperando a ser invitadas.
} 
manifiesta la siguiente persona entrevistada y que constatamos en las observaciones en los bailes, es la existencia de más mujeres que hombres en los bailes: "[...] geralmente você tem muito mais mulheres que vão à milonga ${ }^{4}$ e geralmente quem define a dança é o homem. Eu tenho, eu sempre tive dificuldade com isso, então eu sempre convidei os caras pra dançar" (PERSONA ENTREVISTADA 1). Lo que manifiesta la entrevistada ya se realiza en bailes de tango que no son tradicionales, cuando se entra en estos bailes ya se les avisa a las personas que no se sigue un código de cabeceo tradicional en el que los hombres invitan y las mujeres tienen que esperar a ser invitadas. En la ciudad de Montevideo por lo que se observó en los bailes y lo que manifiestan las personas entrevistas no es tan usado el cabeceo. El cabeceo es uno de los códigos más cuestionados y que es visto como una de las expresiones machistas del tango, porque proporciona el poder de los hombres a elegir solo a ellos con quien bailar y a las mujeres a un rol pasivo de espera.

Como manifiestan Yamanashi y Bulman (2007) los hombres tienen más libertad y son mostrados en los programas televisivos como los que tienen que conducir a la mujer en el baile, mostrándolos como el líder asociado a un estereotipo que tiene que asumir ciertas actitudes de cierta masculinidad y la mujer como pasiva, elegante y frágil que tiene que esperar a ser invitada a bailar y bailar siendo conducida en el baile por un hombre (YAMANASHI; BULMAN, 2007). En este caso la persona entrevistada 1 no sigue ese mandato patriarcal y quiebra esa tradición inventada (HOBSBAWM; RANGER, 2012) invitando a bailar a los hombres siendo mujer.

Otra de las personas entrevistadas habla de esos códigos del tango que se ablandaron en algunos lugares de baile y cuenta la experiencia vivida por una mujer en un baile: "[...] en donde todos estos códigos pasan, no a desaparecer porque en definitiva son códigos que tienen que ver con las relaciones humanas, pero si a ablandarse y a tranquilizarse, para mucha gente fue como un bálsamo viste, que venga una señora milonguera tradicional, a bailar pueda sentarse en una mesa y en determinada tanda ser ella la que invita a bailar" (PERSONA ENTREVISTADA 2). Lo que puede observarse de este relato es que la danza como expresión de ocio puede provocar cambios en las personas, como manifiesta la persona entrevistada 2, ese ablandarse de los códigos provoca la satisfacción en las personas, que puede ser que posiblemente vivenciaron ir a bailar esperando a que sean invitadas, bailando poco en algunas ocasiones por culpa de este código y ahora se sienten empoderadas para poder ellas invitar a bailar.

Shaw (2001) va a decir que el ocio de resistencia empodera a las personas y puede brindar o ser un puente para cambios sociales positivos y ese cambio puede ser individual y/o colectivo. En este caso las personas que vienen investigando y proponiendo otros espacios de aprendizaje y/o baile queer/gay/libre, vienen a brindar un lugar para el encuentro de las personas que no se identifican con ciertos estereotipos de género que existen en la danza de tango y samba gafieira (CECCONI, 2009).

En relación a las clases y bailes presenciados, se observa que hay una parte de las personas que actúan dando clases que tienen en cuenta la enseñanza de la danza desde una perspectiva no heteronormativa y que planifican sus clases teniendo en cuenta la diversidad de posibilidades y formas de expresión del cuerpo en las danzas de salón. En algunas clases observadas se percibió que no existe más la predominancia heteronormativa, habilitando diferentes formas de bailar, donde la persona que conduce en la danza no está en función de su sexo, las personas van a aprender y bailar con vestimentas que no se ajustan a un código o norma no específica, hay hombres que van

\footnotetext{
${ }^{4}$ Milonga es como se denomina muchas veces el baile de tango, asimismo es una danza de salón.
} 
a bailar con zapatos de taco y pollera, mujeres que van de pantalón social, se observa una estética de baile diferente que en los lugares tradicionales de baile de salón y se observan menos estereotipos de género que en los lugares tradicionales.

Una de las personas entrevistadas manifiesta las diferencias que suceden en los espacios de baile queer en relación a los espacios tradicionales: "[...] lo que pasa en la queer es que no corre tanto el estereotipo no es que las mujeres van de vestido y zapatos, van cada uno como quiere, hay una cuestión del calzado que se tiene que mantener por el tango en sí, pero lo que se rompe es el estereotipo del hombre y de la mujer, de la mujer que va de pollera y el hombre de pantalón, sino que por ahí hay hombres de polleras" (PERSONA ENTREVISTADA 5). Estos cambios que manifiesta la persona entrevistada 5 son notorios en relación con las milongas tradicionales de tango y hablan de un espacio queer disponible que desafía los estereotipos de género y de vestimenta.

La resistencia a ciertos estereotipos de género y/o discriminaciones se visibiliza de diversas formas: continuando bailando en el caso de algún tipo de discriminación verbal, ir a bailar a espacios públicos para dar una mayor visibilidad, evitando lugares tradicionales de danza de salón, frecuentando lugares y clases en los que existe mayor apertura (queer, gay, libres) y no son tan tradicionales.

Las personas entrevistadas en Buenos Aires, Montevideo y São Paulo manifiestan que existen miradas de desacuerdo todavía en algunas milongas tradicionales, este tipo de discriminación no explícito, como puede ser un cartel, va desde las miradas de desaprobación que sienten las personas por bailar con una persona del mismo sexo biológico. También existen ciertas miradas de desaprobación que sienten las personas en ciertas milongas tradicionales por la ropa que utilizan cuando van a bailar. Esas miradas se posicionan como un tipo de violencia no verbal que es sentida por las personas que cuestionan los códigos y ciertos estereotipos de género, esa mirada de desaprobación puede ser tan violenta como un insulto o una agresión física.

Dos de las personas entrevistadas manifiestan que sufrieron en algún momento dentro de un lugar de baile de danza de salón comentarios en voz alta como "[...] el tango es para machos" (PERSONA ENTREVISTADA 2), "[...] habiendo tanta mujeres lindas no entiendo porque bailan entre hombres" (PERSONA ENTREVISTADA 4). Este tipo de violencia verbal que quiere imponer la norma heteronormativa es realizada con impunidad por parte de personas en un baile social.

Se observa que las personas que en algún momento sufrieron algún tipo de discriminación por bailar con otra persona del mismo sexo biológico, manifestaron mayoritariamente que nunca reaccionaron a esas discriminaciones, continuaron bailando haciendo caso omiso de la discriminación, su forma de resistir a la agresión fue seguir bailando.

Una de las personas entrevistadas expresa de cómo algunos hombres heterosexuales se sienten ofendidos en el baile en cierta medida cuando son invitados a bailar por otro hombre "[...] es preocupante darse cuenta de que un cara, un hombre hetero xis, bailarino de tango se ve tan atingido, ofendido o, como se dice cuando una persona, presionada, se senté disgustosa" (PERSONA ENTREVISTADA 6). Lo que puede ser analizado como una resistencia también de ciertos grupos tradicionales o conservadores a perder la hegemonía. Butler, Gambetti y Sabsay (2016) van a manifestar que existe una cierta manifestación de que los grupos tradicionales y/o conservadores se sienten vulnerables a los cambios, porque una persona puede decir que no si es invitada a bailar, lo que llama la atención en el relato y es preocupante como bien dice la persona entrevistada 6 , es que se sienta ofendido ese hombre por ser invitado en un baile a bailar por otro hombre. Pareciera por otro lado que ese disgusto 
que sienten algunos hombres por ser invitados por otros hombres a bailar tiene que ver con el cuestionamiento de una identidad estereotipada como heteronormativa y con un miedo a perder ese lugar de masculinidad y poder (CAMACHO, 2000) que es estereotipado en las danzas de salón.

En algunos bailes de tango y samba gafieira de las tres ciudades observadas, que no son tradicionales, ya se observan cambios en los códigos patriarcales y machistas, estos espacios al no regirse por esos códigos, son lugares en que todas las personas pueden experimentar la danza de salón, son lugares en los que los derechos humanos de las personas están asegurados. Estos espacios están contra el machismo, la discriminación, los estereotipos de género y cuestionan ciertos códigos de baile que forman parte de lo que Hobsbawm y Ranger (2012) denominan una tradición inventada.

En el mismo sentido otra de las cosas que ayudan a dar visibilidad en relación a cuestionar ciertos estereotipos de género, es que en el mundial de tango que se realiza en Buenos Aires y en el que acuden personas de todo el mundo para competir, desde el año 2014 en que se modifica el reglamento, no existe más un rol asignado a un género predeterminado, cualquier persona independiente de su género puede bailar en el rol que quiera. Este cambio en el reglamento del mundial de tango posibilitó dar mayor visibilidad a las personas que quieren bailar por fuera del binomio heteronormativo hombre-mujer.

\section{CONSIDERACIONES}

Se considera que en las danzas de salón de tango y samba gafieira se reproducen mayoritariamente ciertos estereotipos de género que están presentes y son al parecer muy fuertes a la hora de cambiarlos y/o querer cambiarlos. Las danzas de salón demuestran que la heteronormatividad es dominante y que las personas que intentan proponer otra alternativa de baile son muchas veces discriminadas en los lugares tradicionales de baile.

Como manifiesta Shaw (2001) el ocio puede ser relacionado con la autonomía, libertad, auto-expresión, pero también esas atribuciones pueden ser criticadas, en el sentido de que en el ocio se pueden reproducir también roles tradicionales de género, estereotipos de género que están relacionados al poder patriarcal y a relaciones de poder en la sociedad, en este caso relacionados al machismo y la heteronormatividad. Todavía, para Shaw (2001), también existe una resistencia desde el ocio, que puede ser empoderante, y en este caso esa resistencia y empoderamiento es cuestionando la heteronormatividad (BUTLER, 2002) en las danzas de salón, que acompaña los derechos adquiridos por las personas y la comunidad LGBTIQ, lo que posibilita nuevas formas de bailar no heteronormativas, diferentes formas de vestirse, nuevas sensaciones corporales y/o experiencias emancipadoras que pueden vivenciar todas las personas en las danzas de tango y samba gafieira. La danza se constituye como un tiempo-espacio en donde se pueden vivenciar esas resistencias de ocio al poder dominante hegemónico y heteronormativo.

Coincidiendo con lo que afirma Cecconi (2009) los espacios de aprendizaje y baile queer/gay/libres vienen a ofrecer un espacio de danza en el que las personas reinterpretan ciertos estereotipos de la danza de tango y samba gafieira. Concordamos con Cecconi (2009) que estos espacios habilitan que las personas que quieren aprender 
y bailar tango y samba gafieira lo puedan hacer de una manera diferente a los espacios tradicionales o juveniles 5 .

En este sentido son fundamentales los espacios de aprendizaje de danza de salón, como manifiesta Risner (2002), que no son heteronormativos, que no son homofóbicos y donde se respeten la orientación sexual de las personas.

Por otro lado, como manifiestan Butler, Gambetti y Sabsay (2016) se percibe que los grupos tradicionales de danza de salón heteronormativos, desde el poder hegemónico, se colocan como grupos vulnerables, víctimas de los cambios sociales y resisten a los cambios, como también manifiestan Jessup, Bundy y Cornell (2013) perpetuando un discurso hegemónico heteronormativo.

Jessup, Bundy y Cornell (2013) manifiestan que el ocio puede ser un espacio para la resistencia, pero al mismo tiempo puede ser un espacio donde se perpetúan discursos conservadores, incluso a veces a través de actos de resistencia, podemos relacionarlo con los lugares y escuelas tradicionales de danza de salón y su resistencia a cambiar.

El discurso que quieren perpetuar los grupos conservadores de danza de tango y samba gafieira se puede percibir en las discriminaciones que hacen las personas cuando gritan en voz alta "el tango es para machos", en las miradas de desaprobación que las personas realizan cuando ven bailar a persona del mismo sexo, en la incomodidad/ofensa que parecen sentir algunas personas heterosexuales por ver bailar a dos personas del mismo sexo, en las prohibiciones explícitas o implícitas que hasta el día de hoy siguen presentes en algunos bailes de las tres ciudades observadas en relación a que dos personas del mismo sexo bailen juntas, en el uso de ciertos códigos de baile y en la vestimenta heteronormativa.

En la danza de salón se pueden observar las marcas e inscripciones de lo que las personas y la sociedad piensan del cuerpo y la sexualidad. Como manifiesta Lachino (2011) ninguna técnica de danza es neutral, detrás de los ejercicios se ocultan lo que la sociedad piensa sobre el cuerpo, la masculinidad, femineidad y el ser.

Asimismo en la danza de tango y samba gafieira hay personas que se sobreponen a ciertos estereotipos de género, que resisten al machismo y a la homofobia (RISNER, 2007), las personas deciden juntarse para seguir aprendiendo/bailando estas danzas en espacios queer, gay, libre, cuestionando la heteronormatividad hegemónica (CECCONI, 2009), deciden seguir abrazándose con otras personas que no conocían antes, la danza sigue siendo un espacio de encuentro, un espacio para la diversidad sexual, un espacio que respeta la orientación sexual, sigue siendo un espacio para el ocio de resistencia (SHAW, 2001) y un espacio para el empoderamiento de las personas.

\footnotetext{
${ }^{5}$ Cecconi (2009) afirma que existen en Buenos Aires tres tipos de milongas: Tradicionales, Juveniles y Queer. Para la autora, en las tradicionales se reproducen estereotipos heterosexuales de danza y un tipo de vestimenta formal; mientras que en las milongas juveniles sigue mayoritariamente habiendo un estereotipo de danza heterosexual, pero con algunos matices que provocan la apertura a diferentes formas de danzar que no sean heterosexuales solamente, así como su vestimenta es informal mayoritariamente; las milongas queer no se siguen un patrón heterosexual de danza de tango. Se proponen independizar los roles ligados a una identificación sexual o de género de quien baila y su vestimenta es informal.
} 


\section{REFERENCIAS}

ATKINSON, R.; FLINT, J. Accessing hidden and hard-to-reach populations: snowball research strategies. Social Research Update, n. 33, p. 1-4, 2001.

BASSETTI, C. Male dancing body, stigma and normalising processes: playing with (bodily) signifieds/ers of masculinity. Recherches Sociologiques et Anthropologiques, v. 44, n. 2, p. 67-90, 2013.

BEROCAN, F. A dança das regras: a invenção dos estatutos e o lugar de respeito nas gafieiras cariocas. Revista Antropolítica, n. 33, p. 51-71, 2012.

BUENOS AIRES. Ley $\quad$ 5261. Disponible en: http://www2.cedom.gov.ar/es/legislacion/normas/leyes/ley5261.html >. Accedido en: 30 oct. 2018.

BUTLER, J.; GAMBETTI, Z.; SABSAY, L. Vulnerability in resistance. London: Durham and Duke University Press, 2016.

BUTLER, J. Cuerpos que importan: sobre los límites materiales y discursivos del "sexo". Buenos Aires: Paidós, 2002.

CAMACHO, P. Danza y masculinidad: la participación masculina en la danza contemporánea mexicana, dos estudios de caso. México: Cenidi Danza/INBA/CONACULTA, 2000.

CECCONI, S. Tango queer: territorio y performance de una apropiación divergente. Revista Transcultural de Música, n. 13, p. 1-13, 2009.

COLLER, X. Cuadernos metodológicos: estudios de caso. Madrid: Centro de Investigaciones Sociológicas, 2000.

CORBETTA, P. Metodología y técnicas de la investigación social. México: Mc Graw Hill, 2007.

DE LAURETIS, T. Eccentric subjects: feminist theory an historical onsciousness. Feminist studies, v. 16, n. 1, p. 115-150, 1990.

DELGADO, J. M.; GUTIÉRREZ, J. (Orgs.). Métodos y técnicas cualitativas de investigación en ciencias sociales. Madrid: Síntesis, 1999.

FORT I MARRUGAT, O. Cuando danza y género comparten escenario. Ausart Journal for Research in Ar, v. 3, n. 1, p. 54-65, 2015.

FOUCAULT, M. El orden del discurso. Buenos Aires: Tusquets Editores, 1990.

GARD, M. Dancing around the "problem" of boys and dance. Discourse: Studies in the Cultural Politics of Education, v. 22, n. 2, p. 213-225, 2001.

GARRAMUÑO, F. Modernidades primitivas: tango, samba e nação. Belo Horizonte: UFMG, 2009.

GASPARRI, J. Che varón: masculinidades en las letras de tango. Revista Caracol, n. 2, p. 174-215, 2011.

HAVMOELLER, B.; BATCHELlOR, R.; ARAMO, O. The queer tango book - ideas, images and inspiration in the 21st century. Denmark: Birthe Havmoeller/Queertangobook, 2015.

HOBSBAWM, E.; RANGER, T. La invención de la tradición. Barcelona: Crítica, 2012.

JESSUP, G. M.; BUNDY, A. C.; CORNELL, E. To be or to refuse to be? Exploring the concept of leisure as resistance for young people who are visually impaired. Leisure Studies, v. 32, n. 2, p. 191-205, 2013. 
LACHINO, H. La reconquista silenciosa del cuerpo. In: TORTAJADA, M. Danza y género. Ciudad de México: COBAES-DIFOCUR, INBA, 2011. p. 9-12.

MASLAK, M. A.; VOTRUBA, S. "Two to tango": a reflection on gender roles in Argentina. Gender Forum, n. 36, p. 1-8, 2011.

PRECIADO, P. B. Testo yonqui. Madrid: Espasa, 2008.

RISNER, D. Rehearsing masculinity: challenging the "boy code" in dance education. Research in Dance Education, v. 8, n. 2, p. 139-153, 2007.

RISNER, D. Rehearsing heterosexuality: unspoken truths in dance education. Dance Research Journal, v. 34, n. 2, p. 63-78, 2002.

SAIKIN, M. Tango y género: identidades y roles sexuales en el tango argentino. Stuttgart: Abrazos, 2004.

SHAW, S. M. Conceptualizing resistance: women's leisure as political practise. Journal of Leisure Research, v. 33, n. 2, p. 186-201, 2001.

WACQUANT, L. Entre las cuerdas: cuadernos de un aprendiz de boxeador. Buenos Aires: Siglo XXI Editores, 2006.

YAMANASHI, A.; BULMAN, R. The choreography of gender: masculinity, femininity, and the complex dance of identity in the ballroom. Men and Masculinities, v. 11, n. 5, p. 602-621, 2007.

Recibido en: 12 nov. 2018.

Aprobado en: 19 dic. 2018. 